\title{
PREGALACTIC PRODUCTION OF HEAVY ELEMENTS BY QUASARS
}

\author{
SUZY COLLIN \\ Observatoire de Paris-Meudon \\ Place Janssen, 92195 Meudon, France \\ AND \\ JEAN-MARC HURÉ \\ Observatoire de Paris-Meudon \\ Place Janssen, 92195 Meudon, France
}

We address the following questions. Is it possible that the heavy elements observed in high redshift quasars are formed in the vicinity of the black hole? And in this case do quasars produce, mainly during their growing phase, the first heavy elements which enrich the intergalactic medium and the protogalaxies?

\section{Evidences for enrichment and outflows in quasars}

There are now strong evidences for metallicities larger than solar in the central few parsecs of quasars, over a large range of redshifts up to $z \geq 4$. Heavy element abundances deduced in high redshift quasars through the modeling of the Broad Emission Lines (BELs) are at least solar. More impressive even are the overabundances drawn from the modeling of the Broad Absorption Lines (BALs) which can be as high as $100 \mathrm{Z}_{\odot}$. These regions are located at distances of $10^{4} \mathrm{R}_{\mathrm{G}}$ (BELs) and $10^{4}$ to $10^{6} \mathrm{R}_{\mathrm{G}}$ (BALs), i.e. inside the inner few central parsecs of the quasars. BALs trace dense clouds in outflowing winds, with velocities up to a few $10^{4} \mathrm{~km} \mathrm{~s}^{-1}$. From dynamical models one can deduce a rate of outflowing metal mass $\dot{M}_{\text {metals }} \sim$ $10^{-2} Z / Z_{\odot} M_{8} \mathrm{M}_{\odot} \mathrm{yr}^{-1}$, where $M_{8}$ is the mass of the black hole expressed in $10^{8} \mathrm{M}_{\odot}$. This rate corresponds to a kinetic luminosity equal to a few percents of the bolometric luminosity, as expected for objects radiating near their Eddington limit. 


\section{Formation and evolution of stars in the accretion disk}

We propose that this enrichment takes place in the accretion disk fueling the black hole (Collin and Huré 1998, in preparation). To determine the structure of the accretion disk we assume for the inner weakly selfgravitating gravitationally stable region a standard $\alpha$ viscosity (Shakura and Sunayev, 1973, A\&A 24, 337), and for the more distant strongly selfgravitating unstable regions the Lin and Pringle (1987, MNRAS 225, 607) viscosity prescription. We show that the gravitationally unstable regions are dense and molecular. We check that the cooling time scale is very small, even for primordial gas, so star formation is likely to occur. Whatever the masses of the newly formed stars they act as seeds of massive stars by accreting matter from the disk at a high rate, according to a process suggested by Artymowicz, Lin and Wampler (1993, ApJ 409, 592). We verify that the migration time of the stars towards the black hole due to tidal action of the disk is much larger than the accretion time and than the replenishment time (which is small owing to the increased - supersonic - viscosity in the gravitationally unstable region). The migration time is also larger than the evolution time of massive stars, so the process allows the stars to reach the stage of supernovae and explode in the disk. This leads to metal enrichment within a few parsecs from the black hole and provides the outflowing gas, in agreement with what is observed in quasars. This mechanism works as well for small (forming) black holes and for powerful quasars, provided they accrete near the critical rate.

\section{Influence on galaxies and on IGM}

In the course of the process, a fraction of the overabundant gas flows with a high velocity out of the nuclear region and escapes in the forming host galaxy and in the intergalactic medium, enriching them in heavy elements. We propose that this could be the starting point of heavy element formation in the Universe. According to the observed luminosity function of $z>$ 4 quasars and to standard CDM cosmology, we estimate the possible enrichment due to this process, and conclude that it could explain, without the need to appeal to a pregalactic "Population III":

1. the abundances observed in the oldest stars of the Galactic halo, with $Z / Z_{\odot} \sim$ a few $10^{-5}$, assuming that the $210^{6} \mathrm{M}_{\odot}$ black hole observed at the galactic center was formed in the early phase of the Galaxy,

2 . the abundances $\sim 10^{-2}-10^{-1} Z_{\odot}$ observed in high redshift metal and damped $L \alpha$ systems which are supposed to be tracers of massive galaxies, harboring more massive black holes,

3 . the abundances $\sim 10^{-3}-10^{-2} Z_{\odot}$ observed in the intergalactic medium, i.e. in $L \alpha$ forest systems. 